\title{
One blue colour channel or two?
}

\author{
Alexander D Logvinenko $\uparrow$, Sara J Hutchinson§ \\ - Department of Vision Sciences, Glasgow Caledonian University, Glasgow G4 OBA, Scotland, UK; \\ e-mail: A.Logvinenko@gcal.ac.uk; § School of Psychology, Queen's University, Belfast BT9 5BP, \\ Northern Ireland, UK \\ Received 20 October 2003, in revised form 7 October 2004; published online 15 June 2005
}

\begin{abstract}
Contrary to the general belief that the yellow-blue mechanism has lower spatial resolution than the red-green mechanism, it has been recently claimed that both mechanisms have similar spatial sensitivity (McKeefry et al, 2001 Vision Research 41 245-255). Studying high-spatial-frequency tritanopia (a colour illusion based on spatio-chromatic interactions in human vision), we found strong evidence for the existence of two blue mechanisms - with low and high spatial-frequency resolution. If confirmed, this may resolve the apparent paradox concerning spatial resolution of the yellow-blue mechanism.
\end{abstract}

\section{Introduction}

It is generally established that, although human colour vision begins with excitation of three types of photoreceptors with peak sensitivities in the short (S), middle (M), and long (L) wavelength regions of the spectrum, colour appearance of light is determined by post-receptor mechanisms (eg Wyszecki and Stiles 1982; Kaiser and Boynton 1996). Still, there is no consensus on spectral as well as spatial characteristics of these mechanisms. While they are widely believed to be colour opponent, with at least two chromatic mechanisms existing: yellow-blue (YB) and red-green (RG), there is a growing body of evidence that each of these mechanisms is divided into two unipolar mechanisms: yellow and blue, and red and green (eg Sankeralli and Mullen 2001).

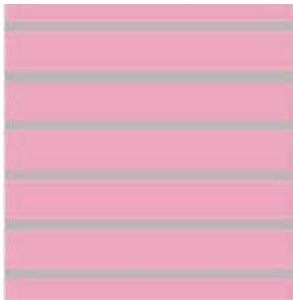

(a)

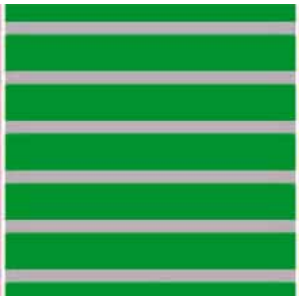

(b)

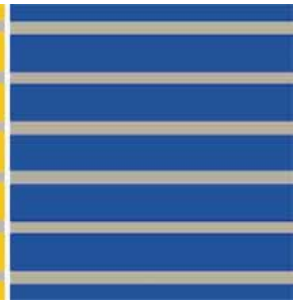

(d)

Figure 1. The high-spatial-frequency tritanopia effect. As viewing distance increases, the achromatic strips in (a), (b), and (c) become coloured blue, yellow, and green respectively. No high-spatialfrequency tritanopia effect is produced from (d) with the achromatic strips remaining neutral as viewing distance increases.

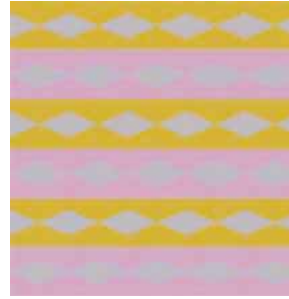

(a)

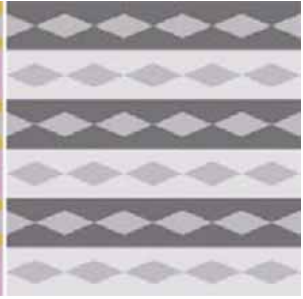

(b)
Figure 2. High-spatial-frequency tritanopia is experienced with (a) but not with its achromatic analogue (b) where $\mathrm{S}$ cone contrast is the same as in (a). 
There is no general agreement concerning spatial characteristics of colour-opponent channels either. Many believe that the YB channel has lower spatial resolution than the RG channel (eg Green 1968; Kelly 1974; Cavonius and Estevez 1975; Hess et al 1989). On the contrary, some authors claimed that the YB channel matches the RG channel in spatial sensitivity (eg Sekiguchi et al 1993; McKeefry et al 2001). We address this issue, using a new approach based on the high-spatial-frequency tritanopia effect (Logvinenko 2001).

If chromatic mechanisms have different spatial characteristics, colour appearance of a pattern would depend not only on its spectral composition but also on its spatial characteristics. Indeed, Wandell and collaborators found that a square-wave grating changes its colour appearance with spatial frequency (Poirson and Wandell 1993; Bauml and Wandell 1996). A similar effect can be observed in figures 1a, 1b, and 1c. As spatial frequency of the grating increases, the grey strips become tinged blue, yellow, or green, depending on the background colour. This colour illusion is called high-spatial-frequency tritanopia, because when the spatial frequency of the grid of achromatic strips in figures $1 \mathrm{a}, 1 \mathrm{~b}$, and $1 \mathrm{c}$ is high enough (it can be achieved either tilting the page with the pictures, or moving it away), it becomes 'invisible' for the $\mathrm{S}$ cone pathway. To be more exact, the patterns in figures $1 \mathrm{a}, 1 \mathrm{~b}$, and $1 \mathrm{c}$ become-in an elegant term of Brainard and Williams (1993) - aliases of homogeneous patterns for the $\mathrm{S}$ cone pathway because it cannot resolve the spatial structure of those patterns. Therefore, observing figures $1 \mathrm{a}, 1 \mathrm{~b}$, and $1 \mathrm{c}$ from a distance, a person with normal trichromatic colour vision would experience what tritanopes (people who supposedly lack $\mathrm{S}$ cones) see in figures $1 \mathrm{a}, 1 \mathrm{~b}$, and $1 \mathrm{c}$ at a close range.

While illusory colours in figure 1 look similar to those observed under tritanopia of the central foveola (Willmer 1944; Mollon 1982), the neural mechanisms of these two colour illusions are probably different. Indeed, tritanopia of the central foveola is usually accounted for by a process of filling-in within the $\mathrm{S}$ cone receptor channel (eg Brainard and Williams 1993; De Valois et al 2000), whereas the high-spatialfrequency tritanopia effect was recently found to be inconsistent with an explanation based on filling-in (Logvinenko 2001). Alternatively, Logvinenko (2001) proposed a filtering-out (spatial mixing) account of high-spatial-frequency tritanopia which suggested that the $\mathrm{S}$ cone coordinate of the strips was mixed with the $\mathrm{S}$ cone coordinate of the diamonds (figure $2 \mathrm{a}$ ), as the $\mathrm{S}$ cone pathway failed to transfer the pattern's contrast.

Furthermore, it was recently shown that high-spatial-frequency tritanopia is a result of malfunctioning of post-receptor mechanisms, rather than a direct consequence of paucity of the $\mathrm{S}$ cones in the retina (Hutchinson 2004; Logvinenko and Hutchinson, in press). Indeed, figure $2 \mathrm{a}$ presents a pattern which invokes quite a strong illusion. However, an achromatic analogue of figure $2 \mathrm{a}$, where the $\mathrm{S}$ components of the diamonds and background, and thus, the $\mathrm{S}$ contrast between the diamonds and background, are exactly the same as in figure $2 \mathrm{a}$, does not produce any colour illusion whatsoever (figure 2b). Therefore, filtering-out must occur within some post-receptor chromatic mechanism. The most likely candidate is the YB mechanism. Indeed, it presumably produces zero response to an achromatic stimulus. Therefore, there is no contrast in terms of the YB mechanism's output between the achromatic diamonds and the achromatic background; thus, no illusion is to be observed. Then, as mentioned above, the YB mechanism is widely believed to have poorer spatial resolution than the $\mathrm{RG}$ and luminance mechanisms. Therefore, the diamonds in figure $2 \mathrm{a}$ may become tinged because the YB mechanism, being unable to resolve contrast between the diamonds and the background, signals the same level of its activation for both the diamonds and the background. As a result, the colour of the diamonds is one which corresponds to the output of the YB mechanism as activated by the background. Observing figure $2 \mathrm{a}$ from a distance allows us to see with our own eyes the response of the YB mechanism to the background. 


\section{Experimental}

Thus, measuring the illusory colour, one can evaluate the responsivity of the mechanism producing the illusion. We have done this for a variety of background colours for two female observers using a Sony colour monitor driven by a PC equipped with VSG 2/5 graphical board. ${ }^{(1)}$ Patterns as in figure $1(5.2 \mathrm{deg} \times 3.2 \mathrm{deg})$ were presented to observers sitting at a distance of $4 \mathrm{~m}$. The vertical distance between the strips and the width of the strips were $0.13 \mathrm{deg}$ (that corresponds to the spatial frequency of 8 cycles $\left.\mathrm{deg}^{-1}\right)$. The width of the strips was a quarter of the distance between them. In the CIE chromaticity diagram, equiluminant $\left(25 \mathrm{~cd} \mathrm{~m}^{-2}\right)$ background colours were chosen at equal intervals along the colour-gamut border. An asymmetric colour-matching task was used to measure illusory colours produced by these background colours. A keypad allowed observers to change the hue, saturation, and intensity of the colour of a matching bar beneath the grating presented on a neutral background $(x=0.314$, $y=0.331$, luminance $=25 \mathrm{~cd} \mathrm{~m}^{-2}$ ).

The chromaticity coordinates of the illusory colours are shown in figures 3 and 4 . Along with yellow and blue, the achromatic strip was tinged with green for some background colours. The green illusory colours were always experienced as less saturated than yellow and blue ones. The illusory green may result from either that the $\mathrm{S}$ cones feed into the RG mechanism, or that the $\mathrm{M}$ and $\mathrm{L}$ receptor channels have different spatial resolution. The position of the illusory colours in the chromaticity diagram resembles the loci of the unique hues (eg Burns et al 1984). Measuring unique hues for our observers showed that unique hues were indeed very close to illusory colours (Hutchinson 2004; Logvinenko and Hutchinson 2004). Therefore, one may assume that it is the chromatic mechanisms which give rise to the unique yellow, blue, and green hues that produce the illusory colours.

It should be noticed that yellow and blue illusory colours are not complementary to each other. Furthermore, illusory blue is always stronger than illusory yellow. For example, it was found that illusory yellow disappeared at 4 cycles $\mathrm{deg}^{-1}$ whereas illusory blue was rather strong at this frequency (Hutchinson 2004; Logvinenko and Hutchinson, submitted). Therefore we are probably dealing with two separate unipolar (yellow and blue) channels with non-overlapping characteristics, rather than one opponent YB mechanism. As mentioned above, such a decomposition into two unipolar (though linear and symmetrical) channels was claimed to take place by other authors (De Valois and De Valois 1993; Sankeralli and Mullen 2001). However, unipolar channels as revealed in the present study are obviously neither linear nor symmetrical.

Note also that not all background colours produce the illusion (marked black in figures 3 and 4). Moreover, some of the background colours which produce no illusion have a quite strong blue component. For example, have a look at figure 1d which produces no illusion at all. If a blue illusory tinge in figure 1a is accounted for by the 'leakage' of the blue component of the background into the strips because of the poor spatial resolution of the YB mechanism, why does such a leakage not take place in figure 1d? A possible answer might be that there are at least two unipolar blue mechanisms, with different spectral and spatial characteristics. If we assume that one of these blue mechanisms, first, has spatial resolution high enough to resolve the contrast of the 8 cycles $\mathrm{deg}^{-1}$ grid as perfectly as the mechanisms driven only by $\mathrm{M}$ and $\mathrm{L}$ cones, and, second, its spectral sensitivity is such that it is virtually insensitive to the background colour in figure 1a, then this can explain why we see strong blue illusory colour in figure $1 \mathrm{a}$ and no illusion in figure $1 \mathrm{~d}$.

(1) A full description of the apparatus, procedure, results and other details can be found elsewhere (Hutchinson 2004; Logvinenko and Hutchinson, submitted). Here we present only a small part of the results relevant to the main subject of this report. 

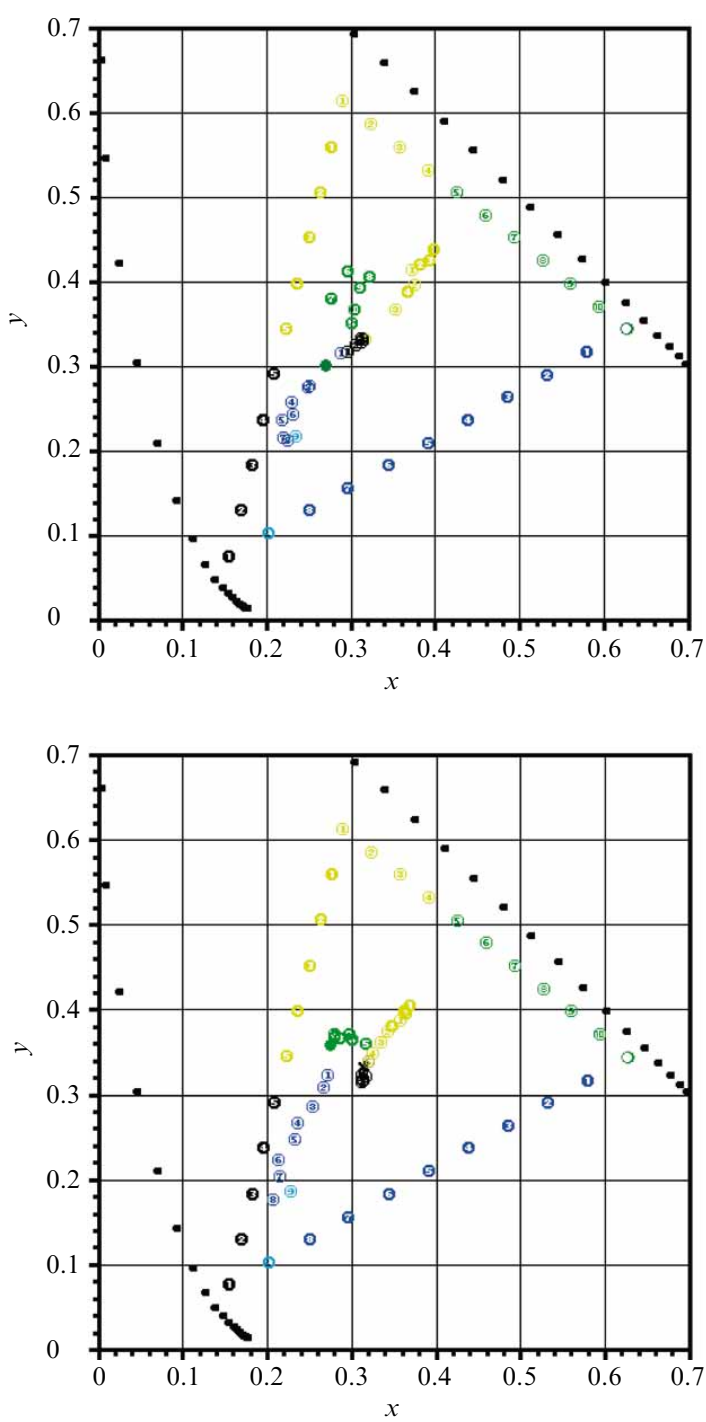

Figure 3. For observer DR, averaged matches obtained with backgrounds varied in the equiluminant plane are presented in the CIE chromaticity diagram. Circles forming the triangle represent the background colours. Circles inside the triangle indicate matches made to illusory colours. Each circle inside the triangle corresponds to a circle on the triangle, indicated by numbers. Colour of circles composing the triangle show the illu sory colour they induce. The black asterisk in the centre stands for the neutral colour. Black circles represent backgrounds that induce nearly no illusion. Small black dots represent the CIE 1931 spectral loci.
Figure 4. Averaged matches for observer SH. Notations are as in figure 3.

Certainly, one needs more data to verify the hypothesis that there are two blue mechanisms, with low and high spatial-frequency resolution. If confirmed, this hypothesis may shed some light on the controversial issue concerning the spatial resolution of the YB channel. Specifically, one may hypothesise that the fine blue mechanism was involved in those experiments where high spatial resolution was established (eg Sekiguchi et al 1993; McKeefry et al 2001), and the coarse blue mechanism was in operation where low spatial resolution was found (eg Green 1968; Kelly 1974; Cavonius and Estevez 1975; Hess et al 1989).

\section{Conclusions}

The idea that there are at least two blue mechanisms, with low and high spatialfrequency resolution, is in line with the claim that multiple spatial-frequency chromatic channels exist (eg De Valois and De Valois 1990, pp 233-235). Furthermore, Humanski and Wilson (1993) claimed that there are three $\mathrm{S}$ cone mechanisms with different spatialfrequency characteristics. Two of the mechanisms are band-pass with peak sensitivities at 0.7 and 1.4 cycles $\mathrm{deg}^{-1}$; the third mechanism is low-pass with a high-frequency cut-off 
at approximately 2.0 cycles $\mathrm{deg}^{-1}$. However, the evidence for the existence of multiple spatial-frequency chromatic channels is very limited, resulting in the issue being controversial. Studying high-spatial-frequency tritanopia provides a good opportunity to revisit this fundamental issue.

Acknowledgment. This work was supported by a research grant from The Wellcome Trust (GR068672MA).

\section{References}

Bauml K, Wandell B A, 1996 "Color appearance of mixture gratings" Vision Research 362849 - 2864 Brainard D H, Williams D R, 1993 "Spatial reconstruction of signals from short-wavelength cones" Vision Research $33105-116$

Burns S A, Elsner A E, Pokorny J, Smith V C, 1984 "The Abney effect: Chromaticity coordinates of unique and other constant hues" Vision Research 24 479-489

Cavonius C R, Estevez O, 1975 "Contrast sensitivity of individual colour mechanisms of human vision" Journal of Physiology 248 649-662

De Valois R L, De Valois K K, 1990 Spatial Vision 2nd edition (New York: Oxford University Press)

De Valois R L, De Valois K K, 1993 “A multi-stage color model” Vision Research 33 1053-1065

De Valois R L, De Valois K K, Mahon L E, 2000 "Contribution of S opponent cells to colour appearance" Proceedings of the National Academy of Sciences $97512-517$

Green D G, 1968 "The contrast sensitivity of the colour mechanisms of the human eye" Journal of Physiology $196415-429$

Hess R F, Mullen K T, Zrenner E, 1989 "Human photopic vision with only short wavelength cones: Post-receptoral properties" Journal of Physiology $417151-172$

Humanski R A, Wilson H R, 1993 "Spatial-frequency adaptation: Evidence for a multiple-channel model of short-wavelength-sensitive-cone spatial vision" Vision Research 33 665-675

Hutchinson S J, 2004 "Colour and shape: Spatio-chromatic interactions in the human visual system", unpublished doctoral dissertation, The Queen's University of Belfast, Belfast, Northern Ireland

Kaiser P K, Boynton R M, 1996 Human Color Vision 2nd edition (Washington, DC: Optical Society of America)

Kelly D H, 1974 "Spatio-temporal frequency characteristics of color-vision mechanisms" Journal of the Optical Society of America $64983-990$

Logvinenko A D, 2001 "High-spatial-frequency tritanopia: S-filling-in or S-filtering-out?" Perception $30223-232$

Logvinenko A D, Hutchinson S J, in press "An effect of luminance contrast on high-spatialfrequency tritanopia" Perception

Logvinenko A D, Hutchinson S J, submitted "Evidence for the existence of colour mechanisms producing unique hues (blue, yellow, and green) as derived from a colour illusion based on spatio-chromatic interactions" Vision Research

McKeefry D J, Murray I J, Kulikowski J J, 2001 "Red-green and blue-yellow mechanisms are matched in sensitivity for temporal and spatial modulation" Vision Research $41245-255$

Mollon J D, 1982 "A taxonomy of tritanopias" Documenta Ophthalmologica Proceedings Series $3387-101$

Poirson A, Wandell B, 1993 "Appearance of colored patterns: Pattern-color separability" Journal of the Optical Society of America A $102458-2470$

Sankeralli M J, Mullen K T, 2001 "Bipolar or rectified chromatic detection mechanisms?" Visual Neuroscience 18127 - 135

Sekiguchi N, Williams D R, Brainard D H, 1993 "Efficiency in detection of isoluminant and isochromatic interference fringes" Journal of the Optical Society of America A 10 2118-2133

Willmer E N, 1944 "Colour of small objects" Nature $153774-775$

Wyszecki G, Stiles W S, 1982 Color Science: Concepts and Methods, Quantitative Data and Formulae 2nd edition (New York: John Wiley and Sons) 


\section{PERCEPTION}

VOLUME 342005

www.perceptionweb.com

Conditions of use. This article may be downloaded from the Perception website for personal research by members of subscribing organisations. Authors are entitled to distribute their own article (in printed form or by e-mail) to up to 50 people. This PDF may not be placed on any website (or other online distribution system) without permission of the publisher. 